\title{
Pulmonary disease caused by Mycobacterium simiae in Iran's national referral center for tuberculosis
}

\author{
Parvaneh Baghaei ${ }^{1}$, Payam Tabarsi ${ }^{1}$, Parissa Farnia ${ }^{2}$, Majid Marjani ${ }^{1}$, Fatemeh-Maryam \\ Sheikholeslami ${ }^{2}$, Mahsa Chitsaz ${ }^{1}$, Pegah Gorji Bayani ${ }^{1}$, Masoud Shamaei ${ }^{1}$, Davood Mansouri ${ }^{1}$, \\ Mohammad Reza Masjedi ${ }^{1}$, Ali Akbar Velayati ${ }^{1}$
}

${ }^{1}$ Clinical Tuberculosis and epidemiology Research Center, NRITLD, Masih Daneshvari Hospital, Shahid Beheshti University of Medical Science and Health Services, Tehran, Iran

${ }^{2}$ Mycobacteriology Research Center, NRITLD, Masih Daneshvari Hospital, Shahid Beheshti University of Medical Science and Health Services, Tehran, Iran

\begin{abstract}
Introduction: Several species of non-tuberculosis mycobacteria (NTM) can affect humans and can cause either symptomatic or asymptomatic infection. This study aimed to determine the clinical and radiological manifestation, as well as the treatment, of $M$. simiae in patients in Masih Daneshvari Hospital, a TB referral hospital in Iran.

Methodology: This retrospective study involved all patients presenting to our referral center from 2002 to 2009 , with confirmation of $M$. simiae pulmonary infection. For all patients, sputum smear and culture for identification was performed, as was drug susceptibility testing. Additionally, PCR identification methods for NTM, and high-resolution CT scan were conducted. All patients were treated according to American Thoracic Society recommendations.

Results: In total, 26 cases of $M$. simiae were identified in our center. The mean age of the patients was $58.23 \pm 16.9$ years. Only one patient was HIV positive, and all but one were Iranian. The most frequent symptom was coughing (92.3\%), and 100\% of the patients had nodular lesions. In addition, bronchiectasis and cavitation were present in $84.6 \%$ and $88.5 \%$ respectively. All the patients were resistant to every firstline drug. Two patients failed the treatment, and twenty-four were cured, after which no recurrence of the disease was observed.

Conclusion: $M$. simiae may present with clinical and radiological manifestations consistent with tuberculosis, and be resistant to anti-TB agents. A more efficient treatment for NTMs such as M. simiae is needed, to shorten the period of treatment and proved fewer adverse effects than current therapies.
\end{abstract}

Key words: tuberculosis; NTM; PCR; M. simiae

J Infect Dev Ctries 2012; 6(1):23-28.

(Received 15 June 2010 - Accepted 21 February 2011)

Copyright $(0) 2012$ Baghaei et al. This is an open-access article distributed under the Creative Commons Attribution License, which permits unrestricted use, distribution, and reproduction in any medium, provided the original work is properly cited.

\section{Introduction}

Various types of non-tuberculosis mycobacteria (NTM) have been recognized as a cause of symptomatic or asymptomatic pulmonary infection in humans[1].Increasing rates worldwide of NTM infections in recent decades can be attributed to two major factors: the growing epidemic of HIV infection and the significant improvements in laboratory diagnostic methods $[1,2,3]$.

Mycobacterium simiae, one of the nontuberculous mycobacteria (NTM) that can affect both humans and animals was described as a new species in 1965 when it was isolated from a Macacus rhesus monkey [4]. This bacterium is phylogenetically classified in the same complex as other mycobacteria such as Mycobacterium triplex, Mycobacterium genavense, Mycobacterium heidelbergense, and Mycobacterium lentiflavum [5]. With regard to its chemical and bacteriologic characteristics, it is a slow-growing, rust-colored photochromogenic mycobacterium and is niacin positive. M. simiae infections have been found in various regions such as the southern United States, Israel, and Cuba [6-10]. The infections are most commonly found in immunosuppressed hosts such as HIV-positive individuals, the elderly, children, and those with a history of tuberculosis (TB) [6,11-13]. Furthermore, those with other diagnosed diseases such as diabetes mellitus, cardiovascular disease, and malignancy are more prone to acquiring $M$. simiae infection [11].

$M$. simiae can cause infections in diverse organs, especially in the respiratory system, causing variable 
clinical manifestations. A recent study indicated that individuals of certain ethnic origins, particularly those from the Middle East region, seemed to be more prone to contracting the disease [11].Only a few cases of $M$. simiae infections have been documented, and there is only one established case report of $M$. simiae in Iran [14].

In this particular study, we aimed to determine the clinical and radiological manifestations, as well as the treatment outcome, of patients with $M$. simiae who presented to our referral center in Iran.

\section{Methodology}

Setting

This study was conducted in Masih Daneshvari Hospital, Tehran, Iran, which is the national referral center for tuberculosis and possesses the national reference mycobacteriologic laboratory. Most patients who saw no improvement in their symptoms (such as cough, sputum, fever, weight loss, dyspnea and anorexia) after TB treatment, or the conversion of sputum smear or culture at the end of TB treatment did not happen, were referred to our center from peripheral health centers around the country, with several new cases presenting directly to our institution.

\section{Patient recruitment}

All patients presenting to our center from 2002 to 2009 with confirmed infection with $M$. simiae were included in the study. Sputum smear and culture were performed for all patients. Three cultures were considered for every patient for controlling and confirmation of $M$. simiae.

Identification using Bergey's Manual of Systemic Bacteriology

Sputum inocula produced up to 120 colonies on Lowenstein-Jensen (LJ) slants after incubation for 4 to 8 weeks. Samples which tested negative using polymerase chain-reaction for IS6110 sequences and spoligotyping, both indicating presence of $M$. tuberculosis, were studied further. Bergey's Manual of Systemic Bacteriology [15,16] was used to determine that the specimen in question was atypical Mycobacterium. Conventional biochemical tests for photo induction, niacin production, nitrate reduction, catalase (heat stable and semiquantative), Tween (Roche, Mannheim, Germany) hydrolysis (for a period of 10 days) and urease (Murphy-Hawkins disk method, Merck, Germany) tests were performed [17]. Controls comprising standard negative atypical mycobacterial specimens, obtained from both the Department of Mycobacteriology at the National Institute of Public Health and the Environment of the Netherlands were used. The specimens were also grown on $\mathrm{LJ}$ medium at the temperatures of $25^{\circ} \mathrm{C}$, $37^{\circ} \mathrm{C}, 40^{\circ} \mathrm{C}$, and $45^{\circ} \mathrm{C}$.

\section{Species identification by PCR}

Bacterial DNA was prepared according to van Soolingen et al. [18]. A segment of the $65-\mathrm{kDa}$ heat shock protein gene ( $h s p 65)$ was amplified by specific primers and digested using two restriction enzymes (BstE II and HaeIII) as described by Telenti et al. [19]. Band sizes were estimated and compared to mycobacterial reference strains provided by the American Type Culture Collection or the Collection of the Italian Reference Laboratory for Mycobacteria [20]. Clinical isolates from the Mycobacteriology Laboratory of Verona Hospital were also used [20].

\section{Further testing}

Drug susceptibility testing (DST) was performed on all M. simiae cultures. Culture identification by PCR was done for patients whose infections were resistant to all first-line drugs. Tuberculin skin test (TST) was done with pure protein derivation (PPD), and indurations more than $10 \mathrm{~mm}$ were considered positive. Furthermore, all patients underwent high resolution computed scan (HRCT).

\section{Results}

Twenty-six cases of $M$. simiae infection were identified from among 1,850 patients who were admitted to our centre with suspected tuberculosis between 2002 and 2009. Diagnosis of NTM was based on clinical and microbiological criteria as stated in American Thoracic Society (ATS) guidelines [20].Thirteen patients were female (50\%), and all but one were Iranian (96\%). The mean age of the patients was $58.23 \pm 16.9$ years. Only one patient was HIV positive (4\%).Twenty-one patients $(81 \%)$ had a history of pulmonary illness and treatment, whereas five patients (19\%) were newly presenting cases. Twelve patients (46\%) had concomitant disease. The tuberculin skin test was positive in 20 patients (76.9\%). 
Table 1. Characteristics and demographic factors of the patients

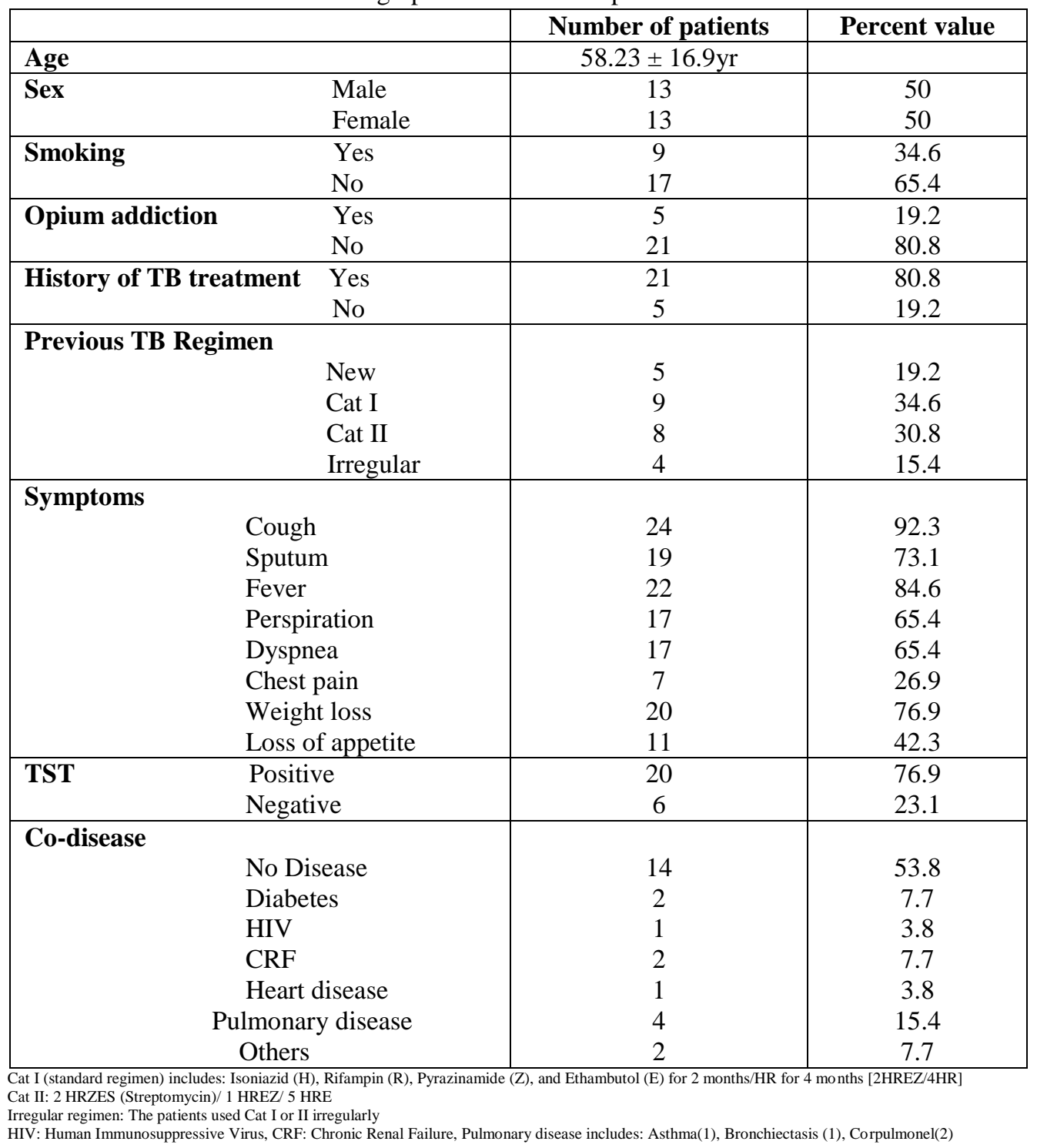

Demographic factors and characteristics factors are summarized in Table 1. Additionally, the clinical manifestations were determined. The most frequent symptom was coughing (92.3\%). All patients had at least one side nodular lesion in HRCT (Table 2).

Drug susceptibility testing was also performed on strains isolated from sputum using all first-line antiTB agents. Interestingly, strains from all patients $(100 \%)$ were resistant to all first-line drugs (isoniazid, rifampin, ethambutol, pyrazinamide, and streptomycin).

All patients with confirmed $M$. simiae infection were treated according to the ATS recommendations on NTM treatment, with a regimen consisting of clarithromycin, ofloxacin, and co-trimoxazole. This regimen was continued for twelve months after culture conversion. After administering the treatment, 24 patients were cured and two patients failed the treatment. There was no recurrence of the disease in any of the patients during two years of follow-up.

\section{Discussion}

After laboratory examination, it was determined that 26 patients were infected with $M$. simiae. These individuals were equally females and males (50\%), between the ages of 17 and 84 , and had a mean age of 58.23 ( \pm 16.9 years). As discussed by Shitrit et al., infection with $M$. simiae is predominantly seen in individuals aged 19 to 87 with a mean age of 44 years [11]. 
Table 2. High resolution computed scan (HRCT) of $M$. simiae infected patients

\begin{tabular}{|c|c|c|c|}
\hline \multicolumn{2}{|c|}{ HRCT Results } & Number of patients & Percent value \\
\hline \multirow[t]{2}{*}{ Nodules } & unilateral & 4 & 15.4 \\
\hline & bilateral & 22 & 84.6 \\
\hline \multirow[t]{3}{*}{ Cavity } & none & 3 & 11.5 \\
\hline & unilateral & 13 & 50 \\
\hline & bilateral & 10 & 38.5 \\
\hline \multicolumn{2}{|c|}{ Bronchiectasis none } & 4 & 15.4 \\
\hline & focal & 9 & 34.6 \\
\hline & multi-focal & 13 & 50 \\
\hline
\end{tabular}

The ATS, on the other hand, reported a mean age of 57 years [21], and their figures closely match the findings in our patients.

In our study, patients infected with $M$. simiae had either been previously diagnosed as being infected with multi-drug resistant tuberculosis (MDR-TB), had received other types of TB treatments, or were new TB cases. Most of our patients were referred to our center after having received prior treatment against $\mathrm{TB}$. Also, all the cases were resistant to all first-line anti-TB agents. As previously established, this reveals that NTMs such as $M$. simiae should be sought meticulously in patients who fail standard anti-TB regimens, and those who are suspected as being infected with MDR-TB [22].

It has also been reported that patients with clinically significant pulmonary diseases are middle-aged or older (55 to 80 years), and either have a history of TB or pre-existing lung abnormalities [3] are prone to M. simiae infection. A study by Maoz et al. showed that underlying conditions or diseases such as smoking, diabetes mellitus (DM), ischemic heart disease (IHD), solid and hematologic malignancies, and chronic obstructive pulmonary disease (COPD) were all associated with $M$. simiae infection [23]. With regard to the medical histories of the patients in this study, it is noteworthy that all but one were HIVnegative. Although the HIV epidemic is suggested as one of the causes for increased NTM infection, Maoz et al. showed that all their 102 patients with $M$. simiae were HIV negative and HIV infection rates were thus significantly lower among $M$. simiae patients compared to M. tuberculosis patients [23]. These results may show that, while other NTMs may be suspected in HIV-positive patients, a clinical suspicion for $M$. simiae infection should also be considered more commonly in non-HIV cases.
Our radiological findings showed that nodular lesion (100\%), cavitation (88.5\%), and bronchiectasis $(84.6 \%)$ are the most common radiographic patterns in our patients. Likewise, in other studies, upper lobe involvement, nodular lesions, and cavitations have been described as the most common radiographic findings [24, 9]. According to Shitrit et al., $38 \%$ of M. simiae patients in their setting had normal chest radiographs. Their study also states a higher occurrence of infection in the upper and middle lobes [11].

Symptoms of the infection that are commonly reported in the literature include sweating, lowgrade fever and weight loss, with coughing, hemoptysis, and sputum production [11] less frequently reported. These statements are consistent with symptoms described by ATS for respiratory NTM infections [21]. In our study, fever and weight loss were common symptoms along with sputum expectoration, dyspnea, and loss of appetite. In addition, almost all our patients suffered from coughing. This is in contrast to the findings by Maoz et al.; in their study, only $17 \%$ of the patients had a cough and this symptom was significantly higher in tuberculosis patients compared to $M$. simiae cases [23].

Our study bears some limitations as well. This study was conducted at Masih Daneshvari Hospital which is a referral center for tuberculosis, meaning that only a subpopulation of $M$. simiae patients presented to our center, mostly those suffering from more serious conditions. There may have been more $M$. simiae cases that were not referred to our center. Furthermore, it is possible for NTMs to infect patients following a previous infection with $M$. tuberculosis. Therefore, it is difficult to determine whether the patient was primarily infected with $M$. simiae or if the present manifestations were the result of $M$. simiae super-infection on the previous tuberculosis. 
Therapy of $M$. simiae pulmonary infection remains an issue. Similar to many other NTMs, most of the isolates are resistant to all first-line antimycobacterial drugs and their response to chemotherapy is variable and is mainly considered as poor [3]. Furthermore, to the best of our knowledge, there have not been any studies on the genotype of $M$. simiae isolates [11]. The patients in our study received a treatment of clarithromycin, ofloxacin, and co-trimoxazole. It is suggested that a three-drug regimen of clarithromycin, moxifloxacin and cotrimoxazole should be administered, as this is known to be successful [3]. Moxifloxacin is not available in Iran. We therefore had to replace moxifloxacin with ofloxacin. We treated our patients according to the American Thoracic Society (ATS) guidelines [21]. Nonetheless, after 24 months follow-up, they all recovered with no recurrence of tuberculosis or other lung disease.

\section{Conclusion}

M. simiae may present with clinical and radiological manifestations consistent with tuberculosis. In most cases, $M$. simiae is resistant to first-line anti-TB medications and therefore anti-TB medication will lead to treatment failure. M. simiae, in contrast to many other NTMs, is frequently seen in HIV-negative patients. Therefore, in cases where a patient fails standard anti-TB treatment, even if HIVnegative, NTMs such as $M$. simiae should be suspected and necessary evaluations should be undertaken.

\section{References}

1. Griffith DE, Aksamit T, Brown-Elliott BA, Catanzaro A, Daley C, Gordin F, Holland SM, Horsburgh R, Huitt G, Iademarco MF, Iseman M, Olivier K, Ruoss S, Von Reyn CF, Wallace RJ Jr, Winthrop K (2003) An Official ATS/IDSA Statement: Diagnosis, Treatment, and Prevention of Nontuberculous Mycobacterial Diseases. Am J Respir Crit Care Med 175: 367-416.

2. Samra Z, Kaufman L, Pitlik S, Bishara J (2005) Emergence of Mycobacterium simiae in respiratory specimens.Scand J Infect Dis 37: 838-841.

3. Piersimoni C and Scarparo C (2008) Pulmonary infections associated with non-tuberculous mycobacteria in immunocompetent patients. Lancet Infect Dis 8: 323-334.

4. Karassova V, Weissfeiler J, Krasznay E (1965) Occurrence of atypical mycobacteria in Macacus rhesus. Acta Microbiol Acad Sci Hung 12: 275-282.

5. Cruz AT, Goytia VK, Starke JR (2007) Mycobacterium simiae Complex Infection in an Immunocompetent child. J Clin Microbiol 45: 2745-2746.

6. Phillips DR, Krishnan H, Watson J (2008) First UK report of successful treatment of Mycobacterium simiae and immune reconstitution inflammatory syndrome in an HIV- seropositive patient. Sex Transm Infect 84: 271-272.

7. Meissner G and Schroder KH (1975) Relationship between Mycobacterium simiae and Mycobacterium habana. Am Rev Respir Dis 111: 196-200.

8. Lavy A and Yoshpe-Purer Y (1982) Isolation of Mycobacterium simiae from clinical specimens in Israel. Tubercle 63:279-285.

9. Bell RC, Higuchi JH, Donovan WN, Krasnow I, Johanson WG Jr (1983) Mycobacterium simiae: Clinical features and follow-up of twenty-four patients. Am Rev Respir Dis 127: 35-38.

10. Valero G, Peters J, Jorgensen JH, Graybill JR (1995) Clinical isolates of Myco bacteriumsimiae in San Antonio, Texas. An 11-year review. Am J Respir Crit Care Med 152: 1555-1557.

11. Shitrit D, Peled N, Bishara J, Priess R, Pitlik S, Samra Z, Mordechai KR (2008) Clinical and radiological features of Mycobacterium kansasii infection and Mycobacterium simiae infection, Respir Med 102: 1598-1603.

12. Patel NC, Minifee PK, Dishop MK, Munoz FM (2007) Mycobacterium simiaecervical lymphadentis. Pediatr Infect Dis J26: 362-363.

13. Braun-Saro B, Esteban J, Jimenez S, Castrillo JM, Fernandez-Guerrero ML (2002) Mycobacterium simiae infection in an immunocompromised patient without acquired immunodeficiency syndrome. Clin Infect Dis 34: E26-27.

14. Mirsaeidi SM, Tabarsi P, Mardanloo A, Ebrahimi G, Amiri M, Farnia P, Sheikhleslami M, Bakayev V, Mohammadi F, Mansouri SD, Masjedi MR, Velayati AA (2006) Pulmonary Mycobacterium simiae infection and HTLV1 infection: an incidental co-infection or a predisposing factor? Monaldi Arch Chest Dis 65: 106-109.

15. Holt JG, Krieg NR, Sneath PHA, Staley JT, William ST (1994) Bergey's Manual of Systemic Bacteriology. $9^{\text {th }}$ edition. Baltimore: Williams and Wilkins 597 p.

16. Kent PT and Kubican GP (1985) Public Health Mycobacteriology: A guide for the level III laboratory, US Department of Health and Human Services, Centers for Diseases Control, Atlanta, GA pp. 36-138.

17. Murphy DB and Hawkins JE (1975) Use of urease test disks in the identification of mycobacteria. J Clin Microbiol 1: 465-468.

18. Van Soolingen D, Hermans PW, de Haas PE, Soll DR, Embden JD (1991) Occurrence and stability of insertion sequences in Mycobacterium tuberculosiscomplex strains: evaluation of an insertion sequence-dependent DNA polymorphism as a tool in the epidemiology of tuberculosis. J Clin Microbiol 29: 2578-2586.

19. Telenti A, Marchesi F, Balz M, Bally F, Bottger EC, Bodmer T (1993) Rapid identification of mycobacteria to the species level by polymerasechain reaction and restriction enzyme analysis. J Clin Microbiol 31: 175-178.

20. Brunello F, Ligozzi M, Cristelli E, Bonora S, Tortoli E, Fontana R (2001) Identification of 54 Mycobacterial species by PCR-Restriction Fragment Length Polymorphism Analysis of the HSP65 Gene. J Clin Microbiol 39: 27992806.

21. Griffith DE, Aksamit T, Brown-Elliott BA, Catanzaro A, Daley C, Gordin F, Holland SM, Horsburgh R, Huitt G, Iademarco MF, Iseman M, Olivier K, Ruoss S, von Reyn CF, Wallace RJ Jr, Winthrop K (2007) An Official ATS/IDSA Statement: diagnosis, treatment, and prevention of nontuberculous mycobacterial diseases. Am J Respir Crit 
Care Med 175: 367-416.

22. Tabarsi P, Baghaei P, Farnia P, Mansouri N, Chitsaz E, Sheikholeslam F, Marjani M, Rouhani N, Mirsaeidi M, Alipanah N, Amiri M, Masjedi MR, Mansouri D (2009) Non-tuberculosis Mycobacteria among patients who are suspected for MDR-TB: Need for earlier identification. Am J Med Sci337: 182-184.

23. Maoz C, Shitrit D, Samra Z, Peled N, Kaufman L, Kramer MR, Bishara J (2008) Pulmonary Mycobacterium simiae infection: comparison with pulmonary tuberculosis. Eur J Clin Microbiol Infect Dis 27: 945-950.

24. Rose HD, Dorff GJ, Lauwasser M, Sheth NK (1982) Pulmonary and disseminated Mycobacterium simiae infection. Am Rev Respir Dis 126: 1110-1113.

\section{Corresponding Author}

Parvaneh Baghaei

Masih Daneshvari Hospital

Darabad, Niavaran Sq

Tehran, Iran 1955841452

Telephone: $+98-21-26109590$

Fax: +98-21-26109590

Email: pbaghaei@gmail.com

Conflict of interests: No conflict of interests is declared. 